\title{
Multi-scale Electrodynamics (MELD): A CAD Tool for Photonics Analysis and Design
}

\author{
R. P. Ratowsky \\ J. S. Kallman \\ R. J. Deri \\ M. D. Pocha
}

February 6, 1998

This is an informal report intended primarily for internal or limited external distribution. The opinions and conclusions stated are those of the author and may or may not be those of the Laboratory.

Work performed under the auspices of the U.S. Department of Energy by the Lawrence Livermore National Laboratory under Contract W-7405-Eng-48. 


\section{DISCLAIMER}

This document was prepared as an account of work sponsored by an agency of the United States Government. Neither the United States Government nor the University of California nor any of their employees, makes any warranty, express or implied, or assumes any legal liability or responsibility for the accuracy, completeness, or usefulness of any information, apparatus, product, or process disclosed, or represents that its use would not infringe privately owned rights. Reference herein to any specific commercial product, process, or service by trade name, trademark, manufacturer, or otherwise, does not necessarily constitute or imply its endorsement, recommendation, or favoring by the United States Government or the University of California. The views and opinions of authors expressed herein do not necessarily state or reflect those of the United States Government or the University of California, and shall not be used for advertising or product endorsement purposes.

This report has been reproduced directly from the best available copy.

Available to DOE and DOE contractors from the Office of Scientific and Technical Information

P.O. Box 62, Oak Ridge, TN 37831

Prices available from (615) 576-8401, FTS 626-8401

Available to the public from the

National Technical Information Service

U.S. Department of Commerce 5285 Port Royal Rd.,

Springfield, VA 22161 


\title{
Multi-scale Electrodynamics (MELD): A CAD Tool for Photonics Analysis and Design
}

\author{
Richard P. Ratowsky and Jeffrey S. Kallman \\ Defense Sciences Engineering Division \\ Electronics Engineering \\ Robert J. Deri and Michael D. Pocha \\ Electronics Engineering Technology Division \\ Electronics Engineering
}

In FY97 we completed work on the (MELD) code, a comprehensive, multiplelength-scale, Graphical User Interface (GUI)-driven photonics design tool. In 1997 MELD was rated one of the one hundred most technologically significant new products of the year by Research and Development magazine.

\section{Introduction}

Photonics circuits using integrated and micro-optic devices are important because they will form the basis of all future high-speed and high-bandwidth communication systems, computers, and signal and image processing hardware. Computational simulation of these devices significantly reduces development and optimization time and cost. However, as photonics devices mature and increase in complexity, designers are faced with components which cannot be modeled using existing methods. A critical reason for this shortcoming is the presence of device feature sizes ranging from subwavelength to thousands of wavelengths, which are not amenable to a single numerical method.

As illustrated in Fig. 1, a photonics device or structure can be placed into one of three classes: optically large; optically small; or optically mixed, meaning large and small-scale features are present in the same device. Optically large components can be treated using beam propagation methods (BPM), which are, however, limited to unidirectional (paraxial) propagation. Optically small components can be modeled using codes which rigorously solve Maxwell's equations (such as finite-difference time-domain (FDTD) methods), to capture wavelength-scale physics. The third class of components (optically mixed) is more difficult to model. If the length scales within the 
component can be decoupled to a good approximation, then hybrid methods using a combination of asymptotic methods and Maxwell solvers in succession may be effective. However, for some problems, a decoupling of length scales is very difficult or impossible.

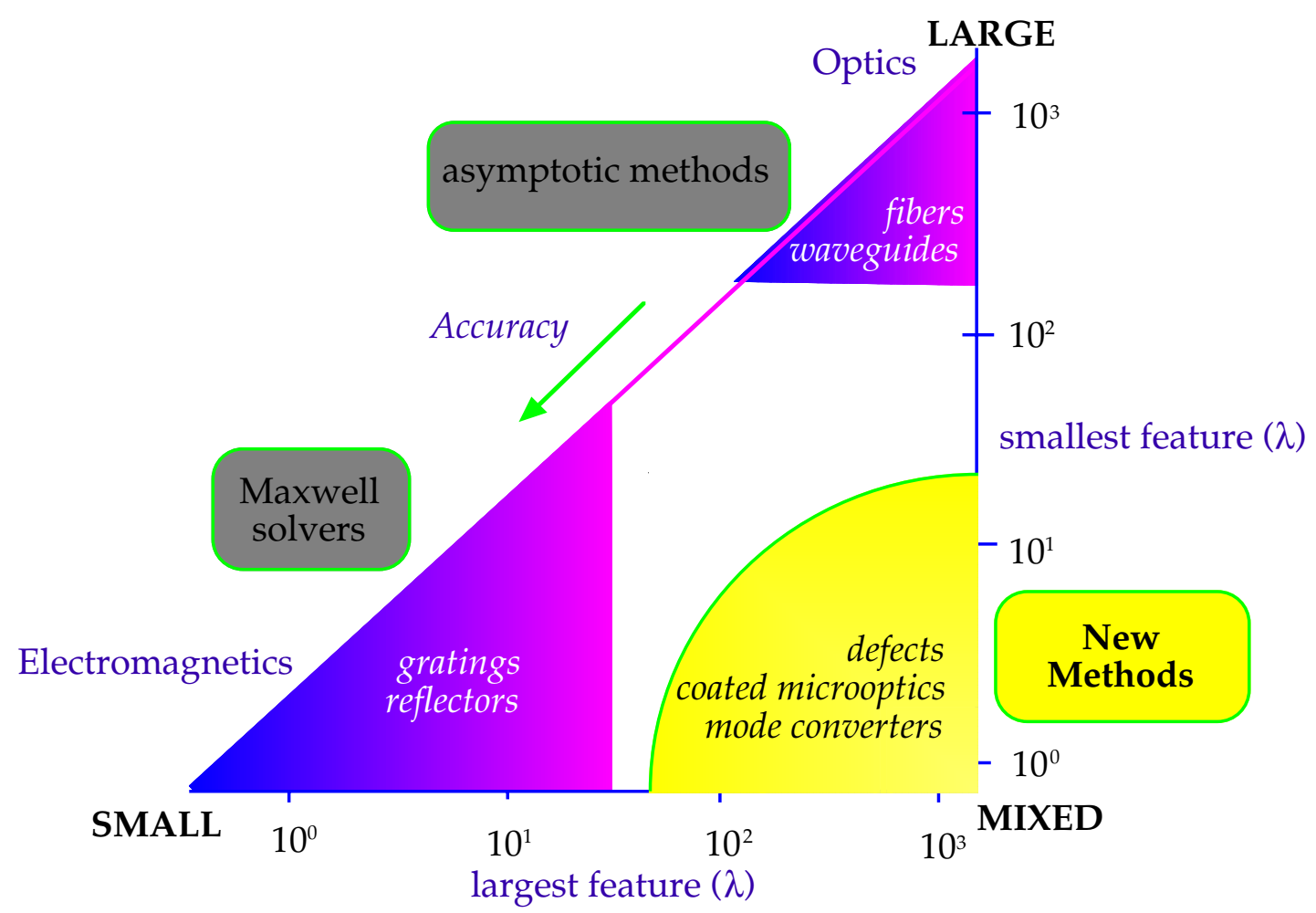

Figure 1. Classification of photonics devices. In terms of largest and smallest feature size, photonics devices can be classified as optically large, optically small, or optically mixed.

To address these issues, the Photonics Analysis and Design LDRD project was proposed in FY96 to create a comprehensive, multiple-lengthscale, Graphical User Interface (GUI) driven photonics design tool, which we call MELD (Multiscale ELectroDynamics). MELD allows the user to compose a 
"virtual optical bench", where micro- or integrated-optical components can be laid out in a modular fashion. Each component is modeled using the most appropriate numerical method, and MELD provides a seamless interface between them.

Simulation in MELD begins when the user assembles an optical bench and "shoots light" from a source (such as a laser diode). Each module has one or more ports which can be connected to ports of other modules. MELD then propagates the light through the system, passing the transmitted field throught the ports from one module to the next while keeping track of the reflected light. The optical field and intensities can be viewed at any port of the system. Fig. 2 shows the MELD 2-d layout for modeling an interferometer system.

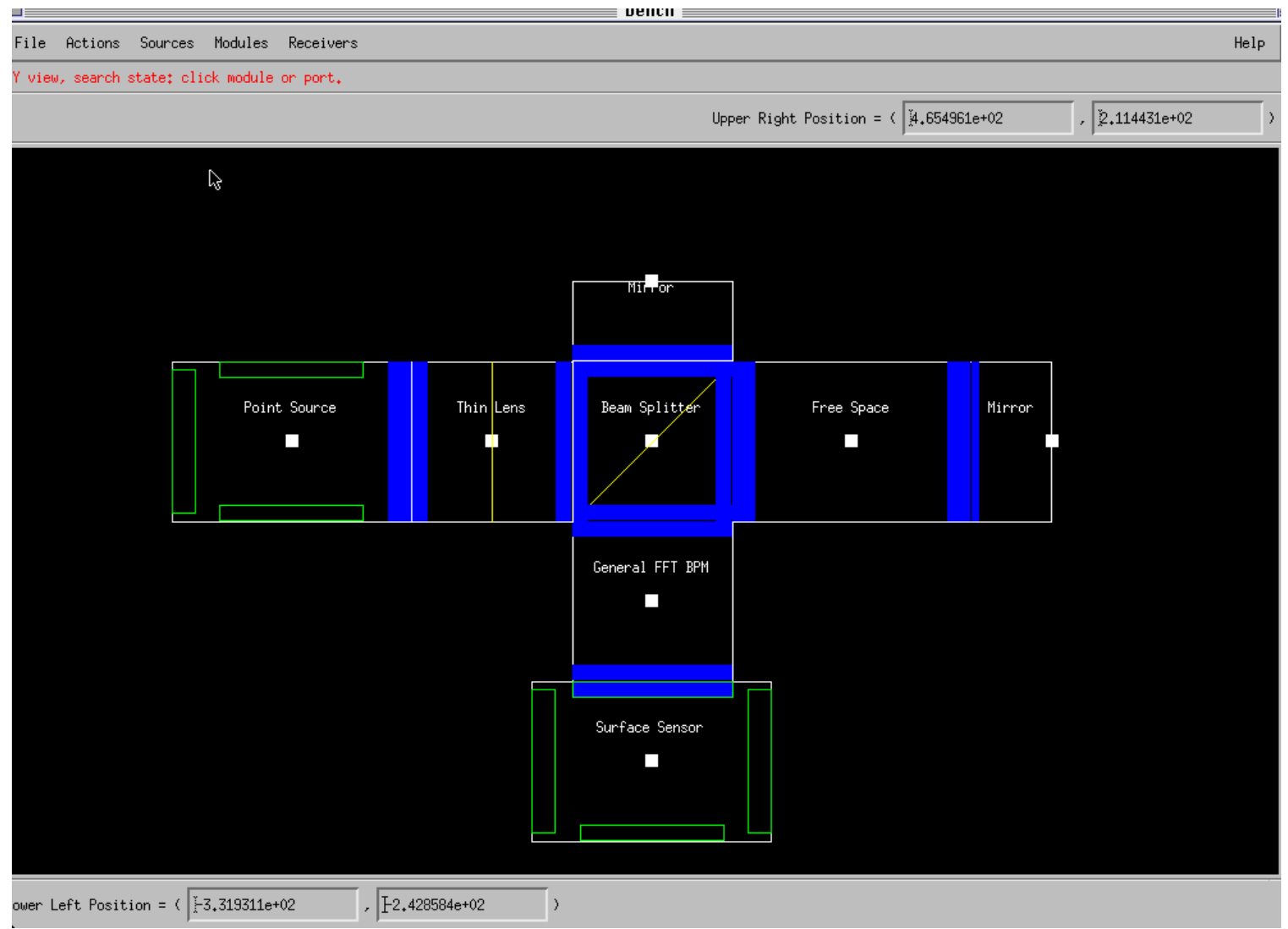

Figure 2. Two-dimensional layout of an interferometer in MELD 


\section{Progress}

In FY97 we made a number of important advances in MELD to bring the LDRD project to its conclusion. These fall into three principal (but overlapping) areas: software, physics, and applications.

Software. We decided to rewrite the bulk of the underlying code using object oriented design in C++("new MELD"). Because of its modular structure, each photonics module in MELD is naturally treated as an object, which allows a great deal of reusable code and greatly simplifies the introduction of new modules. Further, in FY97 we generalized the virtual optical bench to allow two-dimensional layout, thus enabling modeling of important 2D configurations such as Michelson or Mach-Zender interferometers (Fig. 1). The previous version of MELD only allowed uniaxial propagation of light.

We also added optimization and scanning routines in FY97. These allow MELD to perform automatically typical design calculations, such as maximizing coupling efficiency (light throughput) as a function of any system parameter; for example, transverse offset of the source.

A final but essential software accomplishment was documentation. In anticipation of MELD's use outside of our group, we have created a basic user's manual for MELD [1] so the novice user can navigate the program.

Physics. The physics in MELD is embodied in the optical module algorithms. MELD now involves 13 modules: aperture, free space, thin lens, spherical coated lens, coated dielectric plate, directional coupler, mirror, phase plate, beam-splitter, raytrace, general fast Fourier transform (FFT) beam propagation method (BPM) solver, and Finite-Difference-Time-Domain (FDTD) Maxwell Solver, and Transformer. The BPM module solves the Helmholtz equation for optically large waveguiding structures. This module includes a full three-dimensional layout editor (written by us) for the system. 
The transformer is a "passive" module that interpolates fields from one grid to another. Most of these modules were added in FY97. The code framework for the FDTD and ray-trace modules were developed in FY97, but they are not yet fully implemented in the new MELD.

The ray-trace algorithms under development in the MELD project deserve further explication, as they manifest the multiscale spirit through the interface between ray and wave optics. Ray optics is usually a good approximation when the propagation medium is slowly varying on a wavelength scale. However, traditional ray optics cannot capture diffraction at all. In FY97 we developed and implemented an approach to ray optical propagation which allows us to calculate intensity and phase and, within a certain level of approximation, diffaction effects. The motivation for this work is the desire to model optically large elements, possibly with little symmetry, where diffraction effects may be important.

Our approach uses a set of rays distributed in both position and angle called the Wigner distribution [2]. To propagate an optical beam, we first calculate the Wigner distribution from the complex field; it is equal to the Fourier transform of the two-point field correlation function of the field. We then simply propagate the Wigner distribution through the optical system from an input (source) plane to an output plane where we want to calculate the field. (In practice we propagate the rays backwards, since it is more convenient to know where a ray ends up than where it starts). By projecting the output ray distribution in position (nearfield) and angle (farfield), we can recover the phase of the field. This last step is accomplished using an iterative algorithm (Gerchberg-Saxon) which is identical to a technique used in the design of phase plates for NIF [3]. Remarkably, then, we obtain both amplitude and phase information from the field from ray-tracing alone.

We illustrate the accuracy of the method in Fig. 3. Here we show the intensity and phase of a beam which has been propagated through a cylindrical lens using the Wigner method, compared with an "exact" calculation using an expansion in Bessel functions. The agreement is 
excellent, indicating that the method is potentially very valuable for treating aspherical surfaces, which are very difficult to model in any other way. This method is not yet implemented in a MELD module, but will be added in FY98; it is now implemented in a stand-alone GUI-driven program (see Fig. 3).
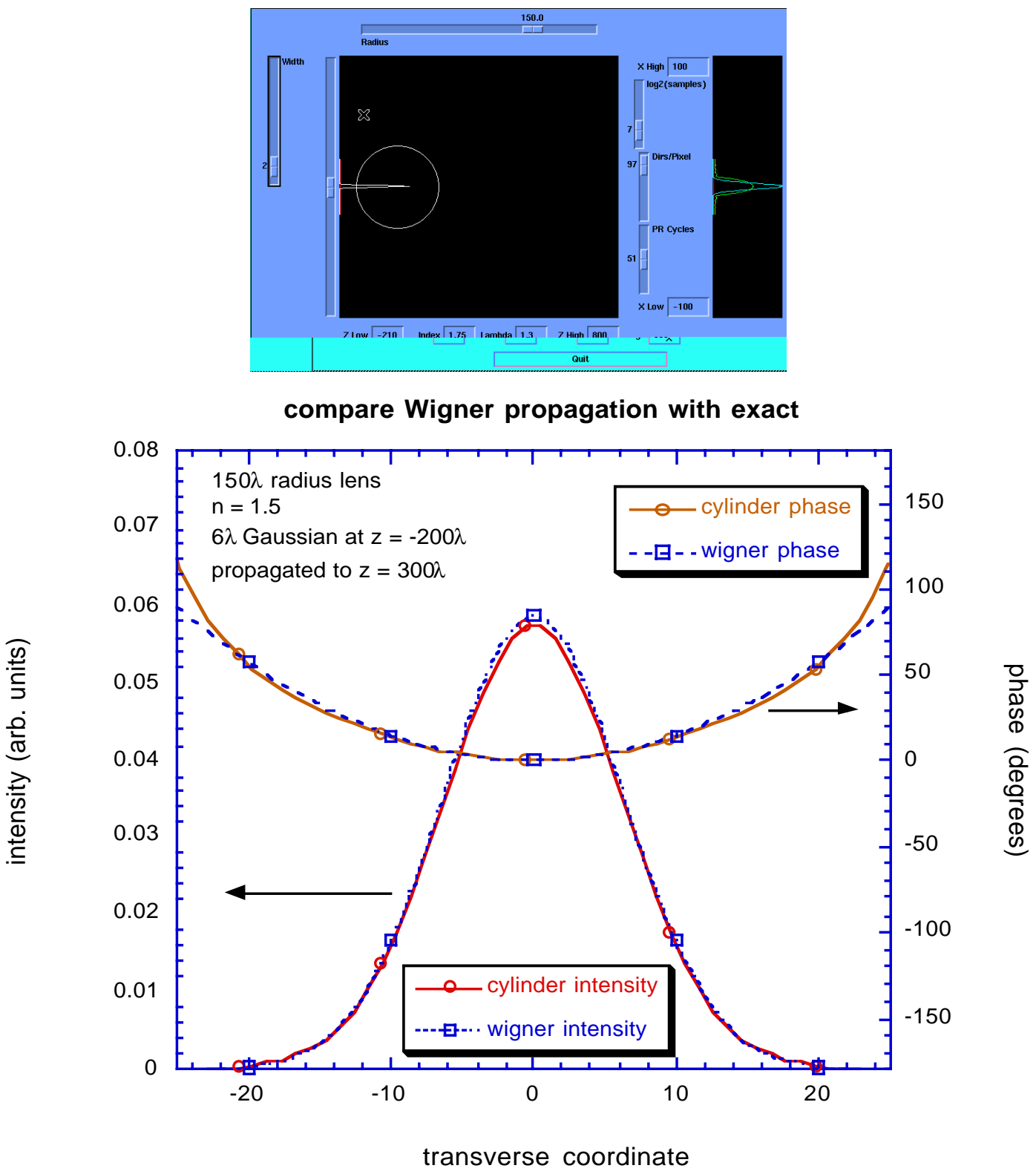

Figure 3. Accuracy of the Wigner method. A beam is propagated through a cylindrical lens very accurately. Curves marked "cylinder" are the exact 
solution. The user interface for the code is also shown.

Applications. We have had an ongoing relationship with HewlettPackard (HP) Laboratories, who continue to use MELD in the design of mode converters. Mode converters are components used to transform the optical mode size in a single-mode device to match that of a dissimilar device, thus increasing the coupling and relieving alignment tolerances. Optimal design of mode converters is an important issue, since a major limitation to the widespread use of photonic systems is the high cost (both in an economic and performance sense) associated with this coupling.

HP has very fruitfully used MELD to design a new mode-converter product line using ball lenses, an example of which is shown in Fig. 2, along with the MELD layout of the device package.
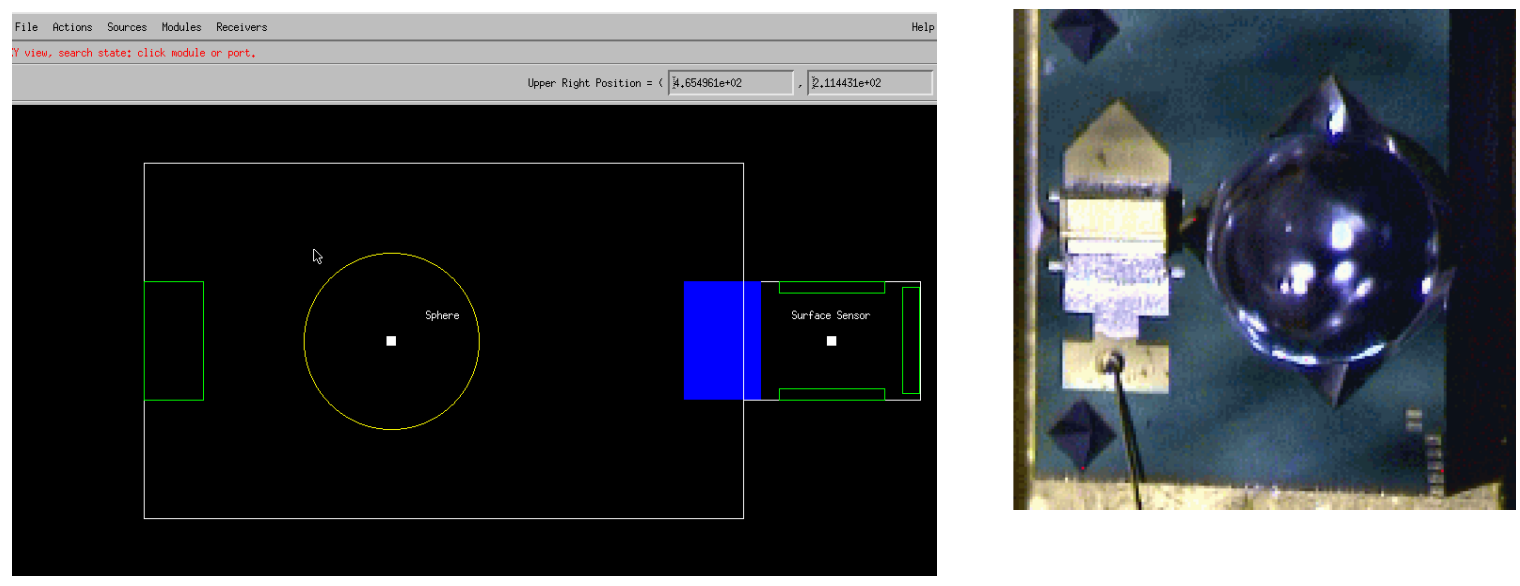

Figure 4. The HP-designed mode-converter package on the right was designed 
using MELD; it includes a laser diode and $750 \mu \mathrm{m}$ diameter ball lens on a silicon submount. On the left is the MELD GUI layout of the device.

We have also created a World Wide Web interface to SPHERE, a subset (stand-alone module) of MELD which can be used for the mode-converter design. In FY97 we also developed the capability to model arbitrary input fields, and this was used by HP to incorporate a realistic model of a novel laser diode design.

We have emphasized the uniqueness of MELD in being able to calculate, very accurately, coupling efficiencies for ball lens mode converters where other commercial software could not [4], and to integrate different length-scale algorithms in a single package. Our results have been recognized through a 1997 R\&D100 award, sponsored by R\&D magazine, as one of the one hundred most technologically significant new products of the year [5].

\section{Future Work}

While the 2-year LDRD effort is now complete, MELD is certainly not a closed-end project, for two reasons. First, the existing code can always be improved; some of the capability we would like to see is not yet implemented to our satisfaction, such as the FDTD solver and the ray-trace module. Second, new modules can and should be added to MELD; their selection will be driven by applications. Furthermore, to be widely accessible the code should be ported to other platforms: MELD currently runs on UNIX workstations using MOTIF graphics. We are exploring options to commercialize the code and fund the additional improvements. 


\section{REFERENCES}

1. J. S. Kallman and R. P. Ratowsky, Introduction to Multiscale ELectroDynamics (MELD), in press.

2. E. Wolf, "Coherence and Radiometry,", J. Opt. Soc. Am. 68, 6-17 (1978); M. Bastiaans, "Application of the Wigner distribution function in optics", in The Wigner Distribution: Theory and Applications to Signal Processing, ed. W.F.G. Mecklenbräuker, North-Holland (1997).

3. S. N. Dixit, M. D. Feit, M. D. Perry and H. T. Powell, “Designing fully continuous phase screens for tailoring focal plane irradiance profiles", Opt. Lett. 21, 1715-17 (1996).

4. R. P. Ratowsky, L. Yang, R. J. Deri, J. S. Kallman and G. Trott," Laser diode to single-mode fiber ball lens coupling efficiency: full-wave calculation and measurements ", Applied Optics 2005, 3435 - 3438 (1997);

R. P. Ratowsky, L. Yang, R. J. Deri, J. S. Kallman and G. Trott,"Ball lens reflections by direct solution of Maxwell's equations", Opt. Lett. 20, 2048 (1995);

L. Yang, K. W. Chang, G. Trott, R. P. Ratowsky, R. J. Deri and J. S. Kallman, "Micro-size ball lenses for micro-optics: theory and experiment" SPIE Photonics West, Jan 1995.

5. "1997 R\&D 100 Awards", Research and Development, September 1997, Vol. 39 (10), p 41. 


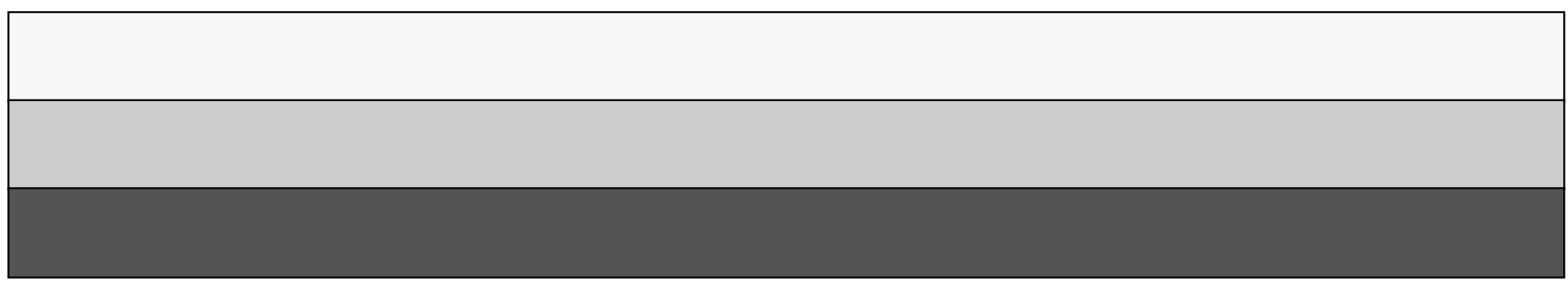

